\title{
ZnO 1-D nanostructures: Low temperature synthesis and characterizations
}

\author{
APURBA DEV, S CHAUDHURI ${ }^{\dagger}$ and B N DEV* \\ Department of Materials Science, Indian Association for the Cultivation of Science, Jadavpur, Kolkata 700 032, India
}

\begin{abstract}
ZnO}$ is one of the most important semiconductors having a wide variety of applications in photonic, field emission and sensing devices. In addition, it exhibits a wide variety of morphologies in the nano regime that can be grown by tuning the growth habit of the $\mathrm{ZnO}$ crystal. Among various nanostructures, oriented 1-D nanoforms are particularly important for applications such as UV laser, sensors, UV LED, field emission displays, piezoelectric nanogenerator etc. We have developed a soft chemical approach to fabricate well-aligned arrays of various 1-D nanoforms like nanonails, nanowires and nanorods. The microstructural and photoluminescence properties of all the structures were investigated and tuned by varying the synthesis parameters. Field emission study from the aligned nanorod arrays exhibited high current density and a low turn-on field. These arrays also exhibited very strong UV emission and week defect emission. These structures can be utilized to fabricate efficient UV LEDs.
\end{abstract}

Keywords. Aligned 1-D ZnO nanostructures; surfactant mediated growth; optical absorption and emission; field emission properties.

\section{Introduction}

Zinc oxide is an outstanding semiconductor having a wide bandgap energy of $3.37 \mathrm{eV}$ and a large excitonic binding energy $(60 \mathrm{meV})$ at room temperature. The excitonic binding energy of $\mathrm{ZnO}$ is much higher than the thermal energy at room temperature $(26 \mathrm{meV})$, and it is also much higher than those of other prospective materials such as ZnSe (22 meV), ZnS (40 meV), and GaN (25 meV), which make it one of the outstanding semiconductors for lasing. The lack of centre of symmetry in wurtzite crystals and large electrochemical coupling result in strong piezoelectric and pyroelectric properties in $\mathrm{ZnO}$ which have important applications like mechanical actuators and piezoelectric sensors (Wang 2004). In addition, $\mathrm{ZnO}$ exhibits a diverse group of growth morphologies in the nano regime that has made this material a promising candidate in the field of nanotechnology. Among the various nanoforms, onedimensional oriented nanostructures such as nanorods, nanowires, nanotubes, nanopins etc are particularly important for efficient field emission that has enormous commercial applications like field emission flat panel displays (Baughman et al 2002), X-ray sources (Senda et al 2004), vacuum microwave amplifiers (Saito and Uemura 2000; Milne et al 2004) etc. With their high melting point, good thermal and chemical stability and low electron affinity (Fancher et al 1998), ZnO one-dimensional

\footnotetext{
*Author for correspondence

(msbnd@iacs.res.in; dev_apurba@yahoo.com)

Since deceased
}

arrays are promising alternatives to carbon nanotubes (CNT) for field emitters with long lifetimes. Moreover, the successful demonstration of UV lasing action (Huang et al 2001) from $\mathrm{ZnO}$ has added a new direction in the field of nanotechnology and motivated subsequent research for the fabrication of one-dimensional $\mathrm{ZnO}$ nanostructured arrays with precise control over size, shape and orientations. The ability to build oriented assemblies of 1-D nanostructures is also very attractive for the fabrication of future photonic (Wang et al 2004; Pan et al 2005), field emission (Li et al 2004) and sensing (Kar et al 2006) devices. As a consequence, several synthetic methodologies have been proposed. Gas phase growth techniques like chemical vapour deposition (CVD), physical vapour deposition (PVD), metalorganic vapour phase epitaxy (MOVPE) and vapour liquid-solid (VLS) etc (Park et al 2002; Lyu et al 2003; Bae et al 2004; Gao and Wang 2004) have been successfully employed to grow $\mathrm{ZnO}$ nanorods and nanowires on solid substrates. However, these methods are very expensive and require high temperature. Recently, solution phase approach to fabricate aligned $\mathrm{ZnO}$ nanorods was utilized (Guo et al 2002; Greene et al 2003; Li et al 2004; Li Q et al 2005; Yu et al 2005; Dev et al 2006). This technique has many advantages as it is cost-effective and large scale-up production is possible. In addition, oriented seed-initiated synthesis (Greene et al 2005) has been found to be very effective to produce highly aligned $\mathrm{ZnO}$ nanorod arrays. This technique (Greene et al 2005) is suitable for preparing long free-standing $\mathrm{ZnO}$ nanorods which exhibit efficient field emission, as long as their aspect ratio is high. But the increase in length is generally accompanied by subsequent increase in diameter. So, if a 
proper approach is adopted to restrict the lateral growth without affecting the orientation of the nanorods they could be expected to exhibit efficient field emission properties for their high aspect ratio and excellent orientation. In addition, the ability to choose crystallographic growth direction of a nanorod array aid in tuning the physical properties of the material, including spontaneous piezoelectric polarization, thermal and electrical conductivity, dielectric constant, lattice strain etc.

In this paper, we report a soft chemical approach to fabricate long and vertically aligned $\mathrm{ZnO}$ nanorod, nanowire and nanonail arrays with a specific crystallographic orientation, on sol-gel derived $\mathrm{ZnO}$ thin films. The role of different experimental parameters was explored with a view to achieve diameter controlled well-aligned $\mathrm{ZnO}$ nanorod arrays.

\section{Experimental}

\subsection{Preparation of $\mathrm{ZnO}$ film}

For the preparation of $\mathrm{ZnO}$ thin films, zinc acetate dihydrate $\left[\mathrm{Zn}\left(\mathrm{CH}_{3} \mathrm{COO}\right)_{2} \cdot 2 \mathrm{H}_{2} \mathrm{O}\right]$ was added in dry ethanol and subsequently a few drops of diethanolamine (DEA) were added with continuous stirring for $1 \mathrm{~h}$. An optimized amount of basic DEA was added to the solution to enhance dissolution of the $\mathrm{Zn}$-salt. Properly cleaned quartz, silicon and glass plates were used as the substrates. The substrates were coated with the sol by a dip coating technique and subsequently dried in an oven at $80^{\circ} \mathrm{C}$ for $5 \mathrm{~min}$. This process was repeated 6 times to increase the film thickness and final films were annealed in air at $600^{\circ} \mathrm{C}$ for $30 \mathrm{~min}$. The entire process was repeated three times again to ensure complete and uniform coverage of $\mathrm{ZnO}$ seeds. Finally, transparent $\mathrm{ZnO}$ films were obtained, which were utilized as the substrates for the formation of $\mathrm{ZnO}$ nanorod arrays.

\subsection{Preparation of $\mathrm{ZnO}$ nanorods}

For the preparation of $\mathrm{ZnO}$ nanorods, $10 \mathrm{mmol}$ of sodium dodecyl sulphate (SDS) and $1 \mathrm{mmol}$ of zinc acetate dihydrate were added to $30 \mathrm{ml}$ of xylene and stirred for $1 \mathrm{~h}$. Then hydrazine hydrate $(80 \%)$ diluted with ethanol was added to the solution very slowly. After $1 \mathrm{~h}$ of stirring, previously prepared transparent $\mathrm{ZnO}$ films were immersed in the solution and refluxed at $90^{\circ} \mathrm{C}$ for $5 \mathrm{~h}$. Finally, the substrates covered with a white layer were washed in distilled water and dried at room temperature. The films thus obtained were used for further characterization.

\subsection{Characterization}

The crystalline phase of the products was determined by X-ray powder diffraction by a Seifert $3000 \mathrm{P}$ diffractometer with $\mathrm{CuK} \alpha$ radiation $(\lambda=1.54 \AA)$. The compositional analysis was done by energy dispersive analysis of $\mathrm{X}$ rays (EDAX, Kevex, Delta Class I). The morphology of the samples was determined by a scanning electron microscope (Hitachi; S-2300). Microstructure and crystal structures of the products were further studied with a high-resolution transmission electron microscope (HRTEM; JEOL 2010). Optical absorbance was recorded by a spectrophotometer (Hitachi, U-3410). Optical absorbance spectra of $\mathrm{ZnO}$ samples (both thin films and nanorod films) were directly measured by placing the films perpendicular to the direction of the incident light. Photoluminescence measurements were carried out with a luminescence spectrometer (Hitachi, FL 2500).
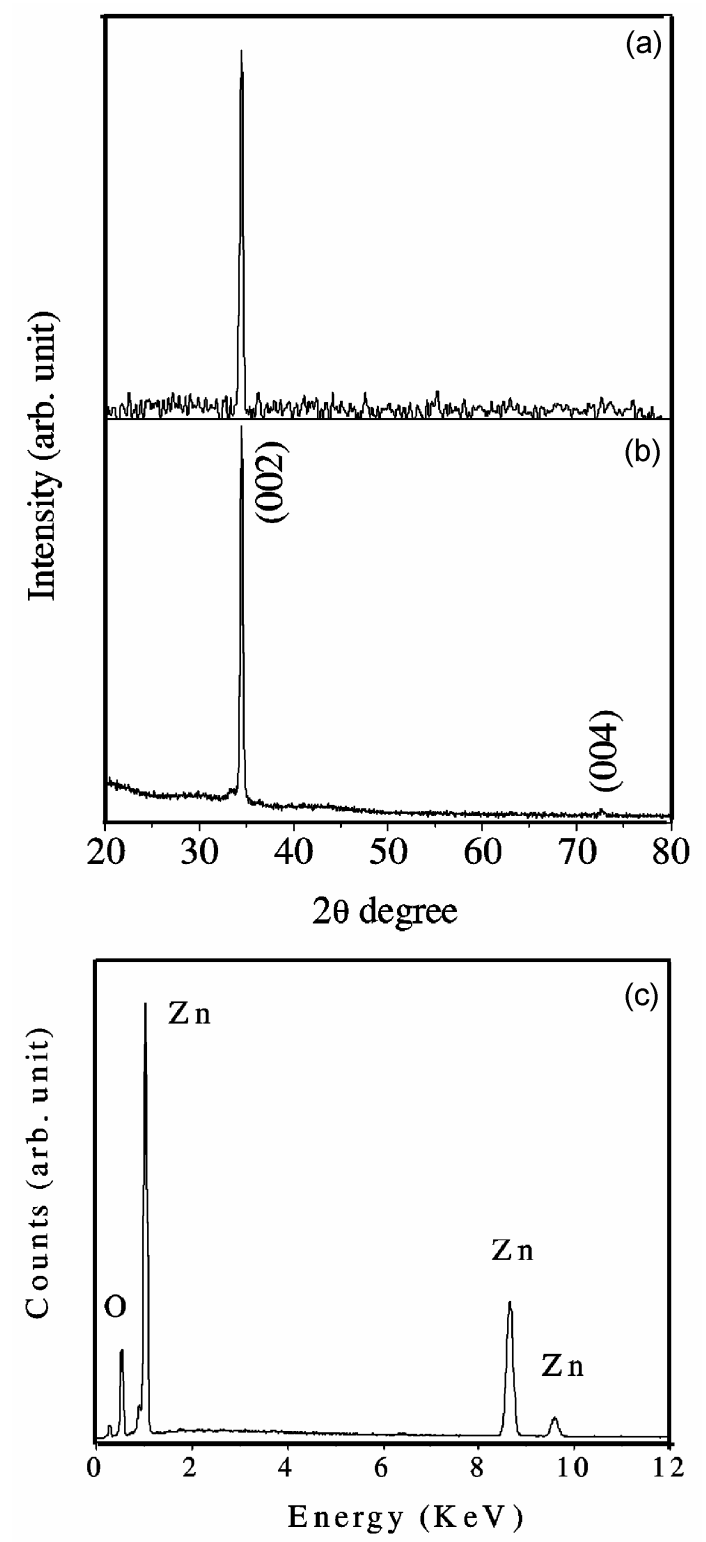

Figure 1. $\mathrm{XRD}$ pattern of (a) $\mathrm{ZnO}$ film on quartz substrate, (b) $\mathrm{ZnO}$ nanorod arrays and (c) EDAX pattern recorded over a representative $\mathrm{ZnO}$ nanorod array. 


\section{Results and discussion}

X-ray diffraction (XRD) was performed on $\mathrm{ZnO}$ films both before and after the formation of nanorods. The $\mathrm{XRD}$ pattern of the transparent $\mathrm{ZnO}$ thin film prior to the nanorod growth is shown in figure 1a. A strong and sharp diffraction peak corresponding to the (002) crystal planes of $\mathrm{ZnO}$, and absence of any other peak, revealed preferred orientation of the film with the $c$-axis of $\mathrm{ZnO}$ crystals aligned along the surface-normal to the substrate. It was observed that the results were independent of the substrates (silicon, quartz and glass) used. The XRD pattern (figure $1 \mathrm{~b}$ ) of the nanorod arrays indicates that the nanorods are well aligned and they grow along the [001] direction following the crystal orientation of the seed. The composition of the nanorods was determined by EDAX study (figure 1c) that revealed the presence of $\mathrm{Zn}$ and $\mathrm{O}$ as the elementary components in stoichiometric amount.

\subsection{Microstructural analysis}

Morphologies and dimensions of the nanorods were studied through SEM and TEM observations. Figure 2(a) shows SEM image of $\mathrm{ZnO}$ thin film revealing uniform distribution of $\mathrm{ZnO}$ seeds on a quartz substrate. Figure 2(b) is the low magnification image of the $\mathrm{ZnO}$ nanorod arrays showing uniformity of the nanorods over a large area. The image in the inset of figure 2 (b) reveals the cross-section of the nanorods to be hexagonal. The image demonstrates that the orientation of the nanorods is quite good and the nanorods grow normal to the substrate. The nanorods have diameters ranging from $80-100 \mathrm{~nm}$ with lengths of $\sim 3 \mu \mathrm{m}$. The magnified tilted view of the nanorods (figure 2c) reveals that the nanorods are well separated from each other.

With a view to investigate the role of the surfactant (SDS) and the base hydrazine hydrate over the dimension and degree of orientation of the $\mathrm{ZnO}$ nanorod arrays, we have carried out a series of experiments and the results are listed in table 1 . $\mathrm{ZnO}$ nanorod arrays were also synthesized without using SDS following identical experimental procedure. It was observed that in all the cases the growth of the nanorods in the lateral dimensions were quite high. The SEM image shown in figure 3(a) reveals that the diameters of the resulting nanorods increased to around $500 \mathrm{~nm}$ within $2 \mathrm{~h}$ of refluxing. Thus these results indicated that the presence of the surfactant (SDS) was essential to regulate the lateral growth of $\mathrm{ZnO}$ nanorods.

Hydrazine hydrate is a strong base and it was used to regulate $\mathrm{pH}$ of the solution. The $\mathrm{pH}$ level of the reaction medium was also varied within a certain range keeping the amount of the surfactant and all other experimental parameters unchanged to investigate its effect on the $\mathrm{ZnO}$ nanorod arrays. Initially, $\mathrm{pH}$ of the solution was measured to be 6.5 when hydrazine hydrate was not added. Experiment was also carried out at this condition keeping other parameters identical and the SEM studies of the resultant film revealed that $\mathrm{ZnO}$ nanorods did not grow on the $\mathrm{ZnO}$ coated substrate. In the next experiment, $\mathrm{pH}$ of the solution was raised to $10 \cdot 6$ by adding proper amount of hydrazine hydrate in the solution, which was then stirred for $1 \mathrm{~h}$ followed by $5 \mathrm{~h}$ refluxing. At this condition uniform growth of aligned and flexible $\mathrm{ZnO}$ nanowires having diameters, $\sim 60 \mathrm{~nm}$ and lengths, $\sim 2 \mu \mathrm{m}$, were observed (figure $3 \mathrm{~b}$ ). With further increase of the $\mathrm{pH}$ of the solu-
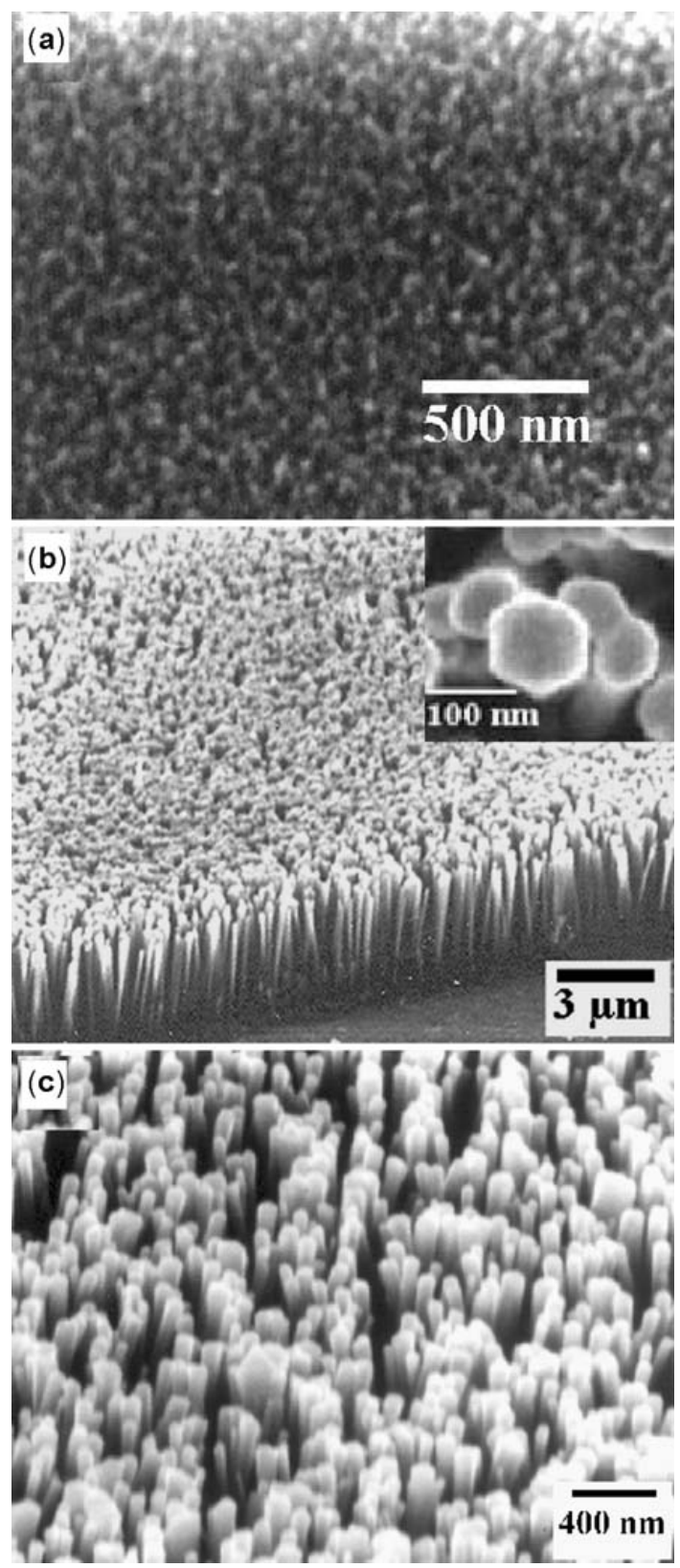

Figure 2. SEM image of (a) $\mathrm{ZnO}$ seed, (b) $\mathrm{ZnO}$ nanorod arrays over a large surface (inset shows the hexagonal tip of the nanorods) and (c) tilted view of the nanorod arrays. 
Table 1. Dependence of nanorod dimensions on $\mathrm{pH}$, surfactant and reaction time.

\begin{tabular}{lccccc}
\hline Sample & $\mathrm{pH}$ of the solution & Surfactant & $\begin{array}{c}\text { Reaction } \\
\text { time }(\mathrm{h})\end{array}$ & $\begin{array}{c}\text { Diameter of the } \\
\text { nanorod }(\mathrm{nm})\end{array}$ & $\begin{array}{c}\text { Length of the } \\
\text { nanorod }(\mu \mathrm{m})\end{array}$ \\
\hline S1 & $10 \cdot 6$ & With SDS & 5 & $\sim 60$ & 2 \\
S2 & $11 \cdot 3$ & With SDS & 5 & $80-100$ & 3 \\
S3 & $11 \cdot 6$ & With SDS & 5 & $\sim 120$ & 6 \\
S4 & $11 \cdot 9$ & With SDS & 3 & $200-250$ & 3 \\
S5 & 11.9 & With SDS & 5 & $200-250$ & 7 \\
S6 & $11 \cdot 6$ & Without SDS & 2 & $\sim 500$ & 2 \\
\hline
\end{tabular}
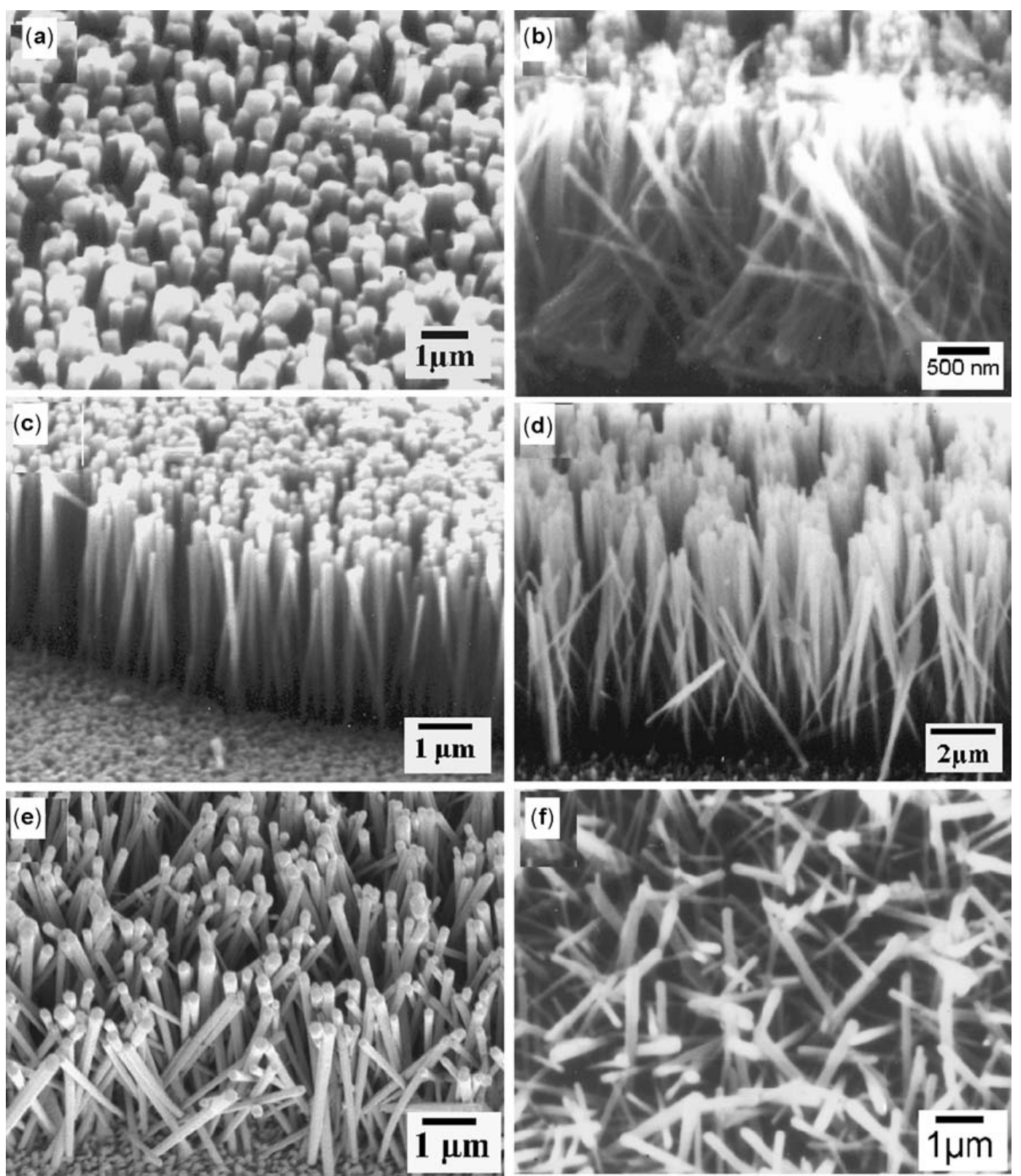

Figure 3. SEM images of nanorods prepared under different experimental conditions as listed in table 1: (a) S6, (b) S1, (c) S2, (d) S3, (e) S4 and (f) S5.

tion to $11 \cdot 3$, the resulting nanoforms were no more wirelike, instead they were rod-like having diameters around 80-100 $\mathrm{nm}$ while lengths increased to $3 \mu \mathrm{m}$ (figure $3 \mathrm{c}$ ).
There was a further increase in length and diameter when the $\mathrm{pH}$ of the solution was increased to $11 \cdot 6$. The SEM image of the sample prepared at this condition is shown 
in figure $3 \mathrm{~d}$ revealing the diameters of the nanorods to be $\sim 120 \mathrm{~nm}$ and lengths, $\sim 6 \mu \mathrm{m}$. It can be observed that the increase in lengths of the nanorods was much higher compared to their diameters with increasing $\mathrm{pH}$ level of the solution. Due to this abrupt increase in the length of the nanorods the freestanding nature of the nanorods were lost and instead they coalesced to each other at their tips. The diameter of the nanorods increased further to around 200-250 nm (figure $3 \mathrm{e}$ ) within $3 \mathrm{~h}$ of refluxing when $\mathrm{pH}$ of the solution was raised to 11.9 , but at this condition orientation of the nanorods collapsed resulting in the nonaligned products (figure $3 \mathrm{e}$ ). A total collapse of the freestanding nature of the nanorods were noticed when the solution was refluxed for $5 \mathrm{~h}$ at this $\mathrm{pH}$ level (figure 3f). Close observation also revealed that the diameter of the nanorods prepared at $\mathrm{pH}$ level 11.6 or higher do not have uniform diameter along their length. The diameter is higher at the top and it is very thin at the bottom resulting in inverted nail like shape.

To investigate the effect of crystallographic orientation of $\mathrm{ZnO}$ thin films on the nanorods arrays, two experiments were carried out using $\mathrm{ZnO}$ thin films having no $c$ axis texturing effect. The $\mathrm{pH}$ of the solution was maintained at 11.3 and 11.6 , respectively. The XRD pattern of $\mathrm{ZnO}$ thin film substrate and the as-prepared $\mathrm{ZnO}$ nanorod

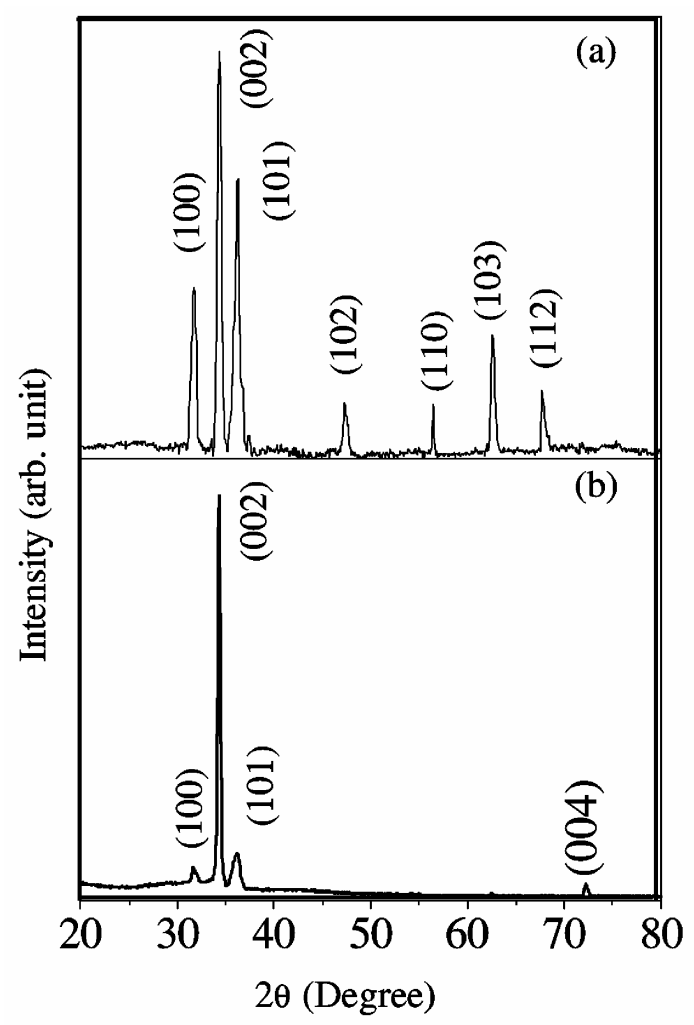

Figure 4. (a) XRD spectra of $\mathrm{ZnO}$ film having no preferred crystallographic orientation and (b) $\mathrm{ZnO}$ nanorod arrays prepared on the above $\mathrm{ZnO}$ film showing enhanced (001) orientation. arrays are shown in figures $4 \mathrm{a}$ and $\mathrm{b}$, respectively. It can be observed that in addition to the diffraction from (002) crystal planes a few more diffraction peaks of $\mathrm{ZnO}$ appeared in the XRD pattern of the $\mathrm{ZnO}$ thin film. However, in the nanorod samples these peaks were quite weak relative to the (002) peak. This could be attributed to the anisotropic growth of $\mathrm{ZnO}$ nanorods along [002] direction. The SEM images corresponding to $\mathrm{pH} 11.3$ and 11.6 are shown in figures $5 \mathrm{a}$ and $\mathrm{b}$, respectively. It can be observed that despite possessing quite identical diameters, the orientations of the nanorods prepared on the non $c$-axis orientated $\mathrm{ZnO}$ thin films (figures $5 \mathrm{a}$ and $\mathrm{b}$ ) were poor compared to the samples, S2 and S3 (figures 3c and d, respectively) prepared with the $c$-axis oriented films under identical experimental conditions.

Transmission electron microscopy (TEM) was also performed to further investigate the size and crystallinity of the $\mathrm{ZnO}$ nanorods. Figure 6a shows the low magnification bright field TEM image of a single nanorod having a length of $\sim 3 \mu \mathrm{m}$. The selected area electron diffraction (SAED) pattern (inset of figure 6a) reveals that the nanorod is single crystalline in nature. The lattice spacing estimated from the high resolution TEM image (figure 6b) of a single nanorod was found to be around $0.26 \mathrm{~nm}$, which

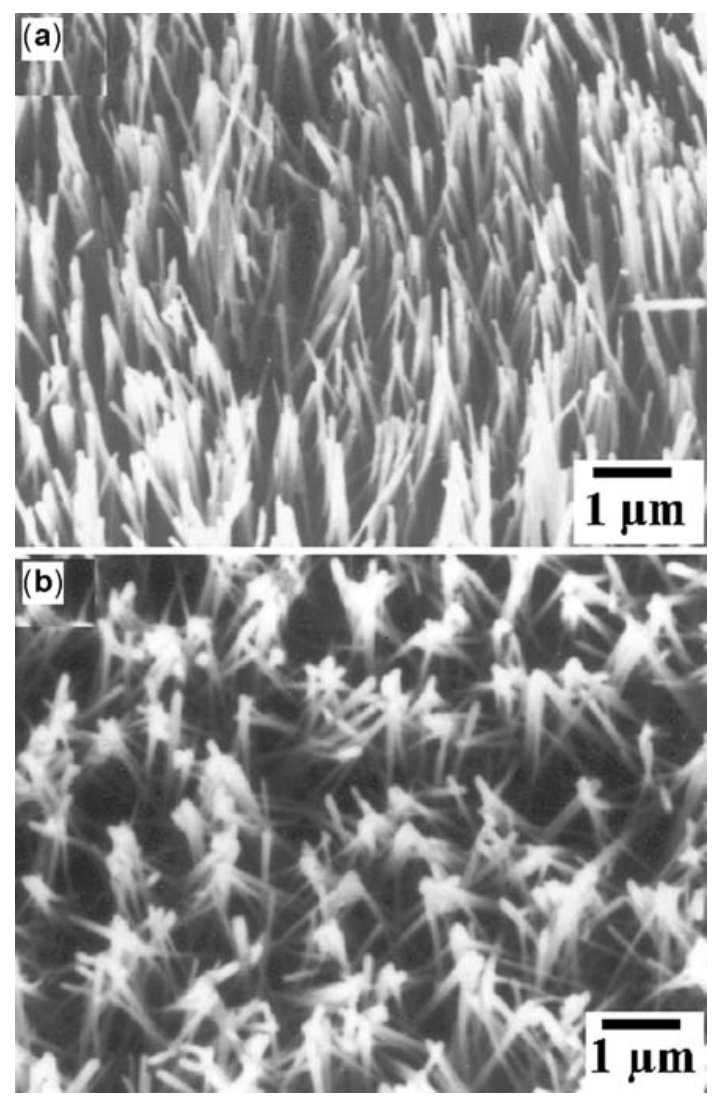

Figure 5. SEM image of the $\mathrm{ZnO}$ nanorod arrays prepared on $\mathrm{ZnO}$ film having no crystallographic orientation at $\mathrm{pH}$ value: (a) 11.3 and (b) 11.6. 

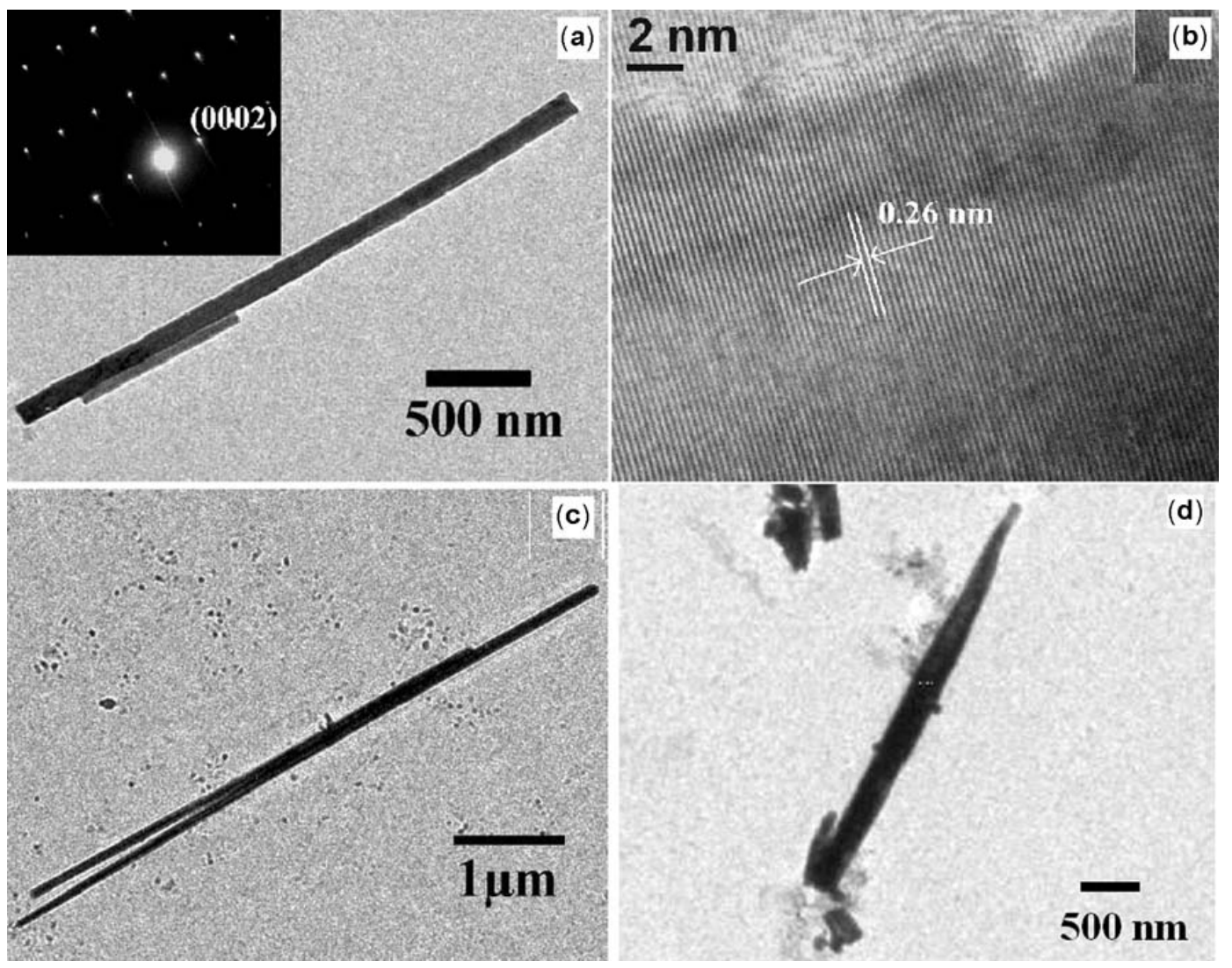

Figure 6. (a) TEM image of a single nanorod with the inset showing corresponding SAED pattern, (b) HRTEM image of a nanorod, (c) TEM image of a long nanorod (S3) and (d) TEM image of a nail like nanoform.

corresponds to the (002) planar spacing of $\mathrm{ZnO}$ in wurtzite phase. Figure $6 \mathrm{c}$ is the TEM image of nanorods formed in higher concentration of hydrazine hydrate (S3). It can be observed that the nanorods have lengths of $\sim 6 \mu \mathrm{m}$ and diameters of $\sim 120 \mathrm{~nm}$. Figure $6 \mathrm{~d}$ shows the TEM image of a nail like nanoform.

\subsection{Growth mechanism}

In growing one-dimensional nanostructures, there are two important steps which control the quality of the products. The first one is the nucleation, which initiates the growth of 1-D nanostructures. In this synthesis process, $c$-axis oriented $\mathrm{ZnO}$ thin films were used. These seeded films served as nucleation sites and also provided a preferential crystallographic growth direction to the resulting nanorods The second step is the growth, and for one-dimensional structure it is important to achieve anisotropic growth with good control over size, shape and orientation. The structure of $\mathrm{ZnO}$ wurtzite crystal can be described as a number of alternating planes composed of tetrahedrally coordinated $\mathrm{O}^{-2}$ and $\mathrm{Zn}^{2+}$ ions, stacked along $c$-axis (Wang 2004). These oppositely charged ions produce positively charged Zn-(001) and negatively charged O-(00-1) sur- faces, resulting in a spontaneous polarization along $c$ axis. Due to this reason, $\mathrm{ZnO}$ surface attracts opposite charges $\left(\mathrm{Zn}^{2+}\right.$ or $\left.\mathrm{OH}^{-}\right)$from the solution forming $\mathrm{Zn}(\mathrm{OH})_{2}$. In this experiment, SDS plays an important role in controlling the lateral growth of the nanorods. The surfactant molecules at a higher concentration are believed to form rod-like micelles (Guo et al 2002; Xiong et al 2002; Lv et al 2004; Zhang et al 2004) which might have formed by entrapping the $\mathrm{Zn}^{2+}$ and $\mathrm{OH}^{-}$ions, already present in the reaction medium, forming rod shaped $\mathrm{Zn}(\mathrm{OH})_{2}$. These rod like micelles are stable at low temperature (Guo et al 2002; Zhang et al 2004) $\left(<100^{\circ} \mathrm{C}\right)$ and during the period of refluxing, the $\mathrm{Zn}(\mathrm{OH})_{2}$ molecules within the micelles begin to decompose. Also, the elevated temperature may have increased the activity of SDS and the micelles to collide with each other and thus increase the length of the nanorods. It can be seen from figures $3 e$ and $f$, that there was an increase in length only, when the period of refluxing was increased from 3-5 h. However, in addition to the above-mentioned mechanism the possibilities of other mechanisms responsible for the restriction of the lateral growth cannot be ruled out. For example, it is also possible that instead of forming rod like micelles, the surfactant molecules get absorbed on the side surface of $\mathrm{ZnO}$ 
nanorods (Sun et al 2002) restricting the lateral growth. The question that remains at this stage is, why should the diameter of the nanorods increase with increasing $\mathrm{pH}$ of the solution. The increase in $\mathrm{pH}$ of the solution leads to the formation of more $\mathrm{Zn}(\mathrm{OH})_{2}$ and thus increase the concentration of $\mathrm{Zn}(\mathrm{OH})_{2}$ in the solution. So, if there exists a concentration difference of $\mathrm{Zn}(\mathrm{OH})_{2}$ between the inside and outside of the rod like micelles, there may be a transfer of $\mathrm{Zn}(\mathrm{OH})_{2}$ from outside to inside the micelles through the surface of the micelles (Xiong et al 2002) and thus increase the diameter of the resulting nanorods. The experiments demonstrated in this paper clearly indicate that the columnar structure of $\mathrm{ZnO}$ could be formed in the solution without SDS (figure $3 a$ ) but the lateral dimension becomes too large as compared to the samples prepared with SDS. So the effect of SDS was to confine lateral growth so that high aspect ratio of the nanorods can be achieved. The synthesis approach described here is also suitable to vary the length of the nanorods within a wide range by using proper amount of hydrazine hydrate.

\subsection{Optical properties}

Figure 7a shows the room temperature optical absorbance spectra of $c$-axis oriented $\mathrm{ZnO}$ thin film and the aligned $\mathrm{ZnO}$ nanorods arrays. A sharp absorption edge at $368 \mathrm{~nm}$ corresponding to $3.37 \mathrm{eV}$ was observed from both the samples. This corresponds to bulk values of the bandgap of $\mathrm{ZnO}$. The absorption study also revealed that the asprepared $\mathrm{ZnO}$ nanorod arrays are transparent in the visible region. Photoluminescence (PL) property of $\mathrm{ZnO}$ was widely investigated for its potential applications in UV photonic devices. This is also an important tool to evaluate crystal defects. The room temperature photoluminescence spectra of the as prepared $\mathrm{ZnO}$ thin film and the nanorod arrays are shown in figure $7 \mathrm{~b}$. The photoluminescence spectra of all the samples show very strong emission at $3.22 \mathrm{eV}$, which is generally attributed to the excitonic emission (Liang et al 2005). It was also observed that the intensity of the excitonic emission depends on the degree of alignment of the $\mathrm{ZnO}$ nanorods. It was observed that the intensity of the UV emission peak was maximum for sample S2 having best alignment and least for the randomly oriented nanorods in sample S5. The intensity of the emission from the $\mathrm{ZnO}$ thin films were quite low compared to the nanorod arrays and the PL plot of the $\mathrm{ZnO}$ thin film was multiplied by a factor for better visibility. It can be observed that in addition to the excitonic emission, the photoluminescence spectrum of $\mathrm{ZnO}$ film consists of a broad emission in the visible green region (figure 7b). This green light emission peak originated due to transition from defect states (Roy et al 2003; Kar et al 2006). However, this defect level emission was very weak in the photoluminescence spectrum of $\mathrm{ZnO}$ nanorods. So it is clear that the defect concentration could be substantially reduced by this synthesis approach. The PL studies of the $\mathrm{ZnO}$ nanorod (Hsu et al 2004), nanowire (Lyu et al 2002) and nanonail (Kar et al 2006) arrays produced by high temperature based thermal evaporation approach revealed that these arrays exhibited a strong defect related emission band along with the excitonic UV emission. Thus $\mathrm{ZnO}$ nanorod arrays having good UV emission property could be synthesized by this surfactant assisted approach.

\subsection{Field emission properties}

For the field emission measurement, the $\mathrm{ZnO}$ nanorod arrays having the best alignment and well-separated
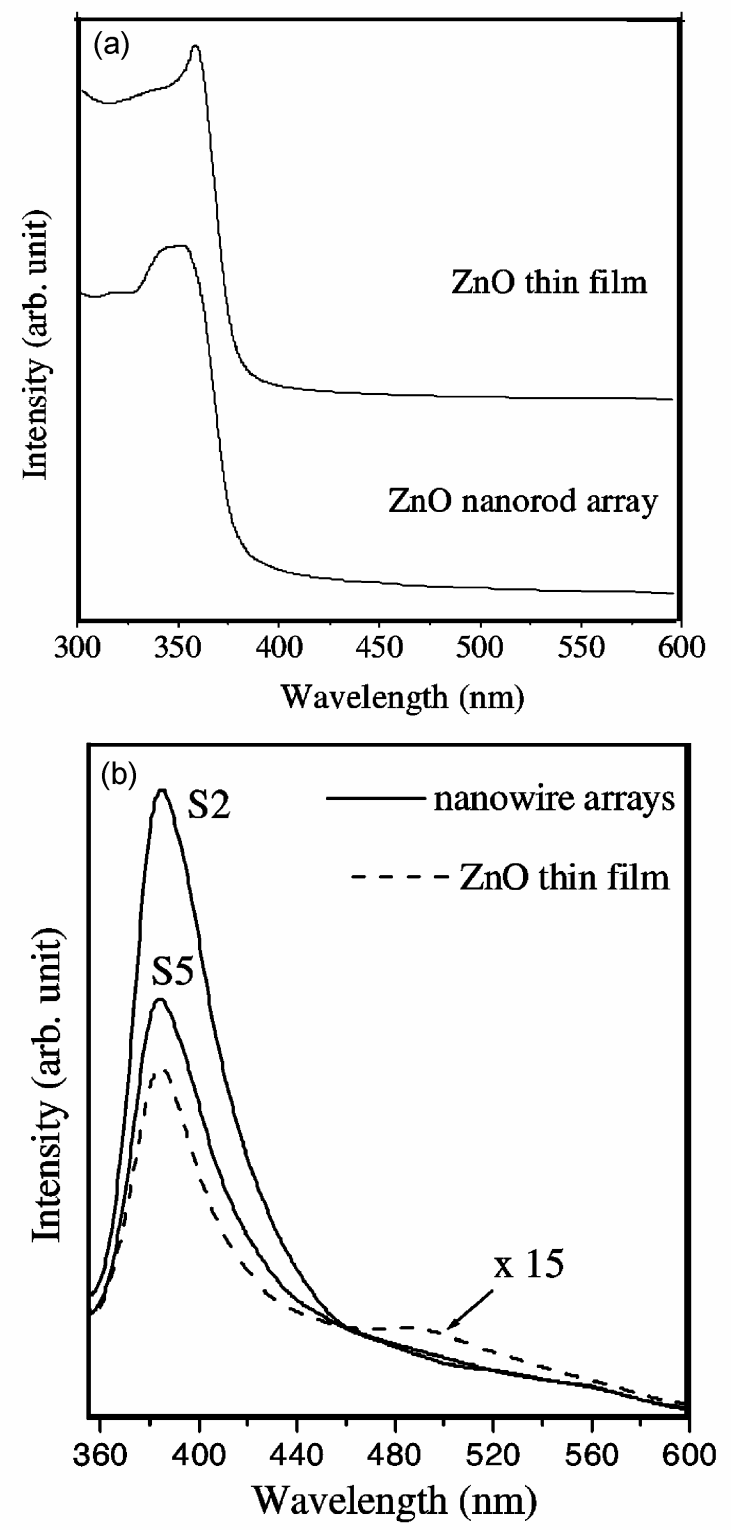

Figure 7. Room temperature optical absorption spectra of (a) the $\mathrm{ZnO}$ thin film with preferred crystallographic orientation and (b) photoluminescence spectra of the $c$-axis oriented $\mathrm{ZnO}$ films before and after the formation of $\mathrm{ZnO}$ nanorod arrays. 
nature (S2) was arranged in two parallel plate configurations in a vacuum chamber with a pressure of $10^{-7}$ Torr and the distance between the electrodes was maintained at $300 \mu \mathrm{m}$. The measured current density as a function of electric field is shown in figure 8a. A very low turn-on electric field of $1.7 \mathrm{~V} / \mu \mathrm{m}$ was obtained at an emission current density of $0 \cdot 1 \mu \mathrm{A} / \mathrm{cm}^{2}$, which reaches to $10 \mu \mathrm{A} / \mathrm{cm}^{2}$ at an electric field of $1.9 \mathrm{~V} / \mu \mathrm{m}$. This value of turn-on electric field is significantly lower than $\mathrm{ZnO}$ nanopencils (Calleja and Cardona 1977), ZnO nanoneedles (Li et al 2004), and $\mathrm{ZnO}$ nanorods coated with amorphous carbon (Liao et al 2005). According to Fowler-Nordheim (F-N) model, the electron emission from a semi-infinite flat metallic surface can be expressed in terms of current density $(J)$ and electric field, $E$, as

$$
J=A\left(\beta^{2} E^{2} / \varphi\right) \exp \left(-B \varphi^{3 / 2} / \beta E\right),
$$
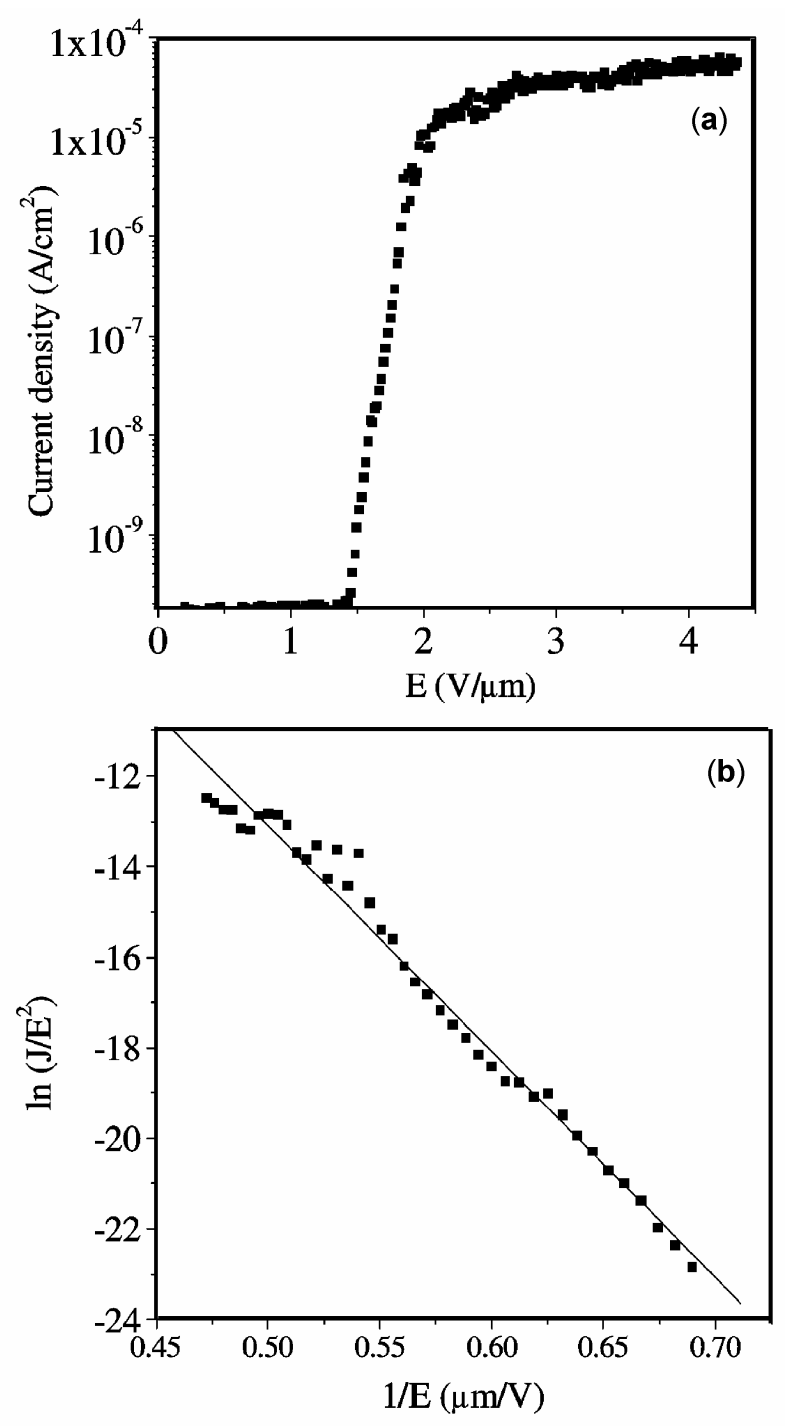

Figure 8. (a) $J-E$ plot of the field emission from the $\mathrm{ZnO}$ nanorod array having best orientation and (b) corresponding $F-N$ plot. where $A$ and $B$ are constants, $A=1.54 \times 10^{-10}\left(\mathrm{AV}^{-2} \mathrm{eV}\right)$, $B=6.83 \times 10^{9}\left(\mathrm{Vm}^{-1} \mathrm{eV}^{-3 / 2}\right)$ and $\varphi$ the work function. $\beta$, the field enhancement factor of the sample, is associated with the magnitude of the electric field at the emitting surface by the relation

$$
E(\text { local })=\beta E,
$$

where $E$ (local) is the local electric field at the emitting top surface. Figure $8 \mathrm{~b}$ shows the plot of $\ln \left(J / E^{2}\right)$ vs $1 / E$, exhibiting linear dependencies, which is in agreement with $(F-N)$ model (eq. (1)). The value of field enhancement factor, $\beta$, can be estimated from the slope of the $(F-N)$ plot if the value of work function, $\varphi$, is known. If we assume the work function $(\varphi)$ as $5.3 \mathrm{eV}$, the value of $\beta$ is calculated to be $\sim 1730$, which is much higher than other recent reports on $\mathrm{ZnO}$ (Cui et al 2005; Ham et al 2005). The field enhancement factor usually depends on the geometry, tip size, length and number density of the nanorods grown on a substrate (Li S Y 2004). For high aerial density of nanorods the local field at the emitting surface reduces due to the electrostatic screening effect provoked by the neighbouring nanorods. On the other hand, long and highly oriented nanorods exhibit much better field emission than short and randomly oriented nanorods (Nilsson et al 2000; Banerjee et al 2004). The vertical growth of the $\mathrm{ZnO}$ nanorods have better ability to enhance local field at the emitting surface and reduce the turn on electric field (Banerjee et al 2004). The excellent field emission property of as-prepared $\mathrm{ZnO}$ nanorod arrays can be attributed to their good orientation and high aspect ratio.

\section{Conclusions}

We have successfully prepared $\mathrm{ZnO}$ thin films with a preferred $c$-axis texturing by sol-gel technique and fabricated well-aligned $\mathrm{ZnO}$ nanorod arrays with tunable length up to $6 \mu \mathrm{m}$ on these $\mathrm{ZnO}$ films by using a simple surfactant-assisted approach. Diameters of the nanorods have been successfully reduced by using surfactant. Wellaligned $\mathrm{ZnO}$ nanorod arrays can be prepared over any substrate so long as a $c$-axis textured uniform $\mathrm{ZnO}$ film can be grown on it. High uniformity of the arrays can also be maintained over a large area by this novel approach. The optical properties of as-prepared nanorod arrays show their good crystalline quality. These films of long and aligned $\mathrm{ZnO}$ nanorods also exhibited very efficient field emission properties with a turn-on field as low as $1.7 \mathrm{~V} / \mu \mathrm{m}$ and a high field enhancement factor $(\beta)$. These films of highly oriented nanorod arrays have potential applications in photonics, field emission displays, nanoelectronics and gas sensing devices.

\section{References}

Bae S Y, Seo H W and Park J 2004 J. Phys. Chem. B108 5206 
Banerjee D, Ho S J and Ren Z F 2004 Adv. Mater. 162028

Baughman R H, Zakhidov A V and de Heer W A 2002 Science 297787

Calleja J M and Cardona M 1977 Phys. Rev. B16 3753

Cui J B, Daghlian C P, Gibson U J, Püsche R, Geithner P and Ley L 2005 J. Appl. Phys. 97044315

Dev A, Kar S, Chakrabarty S and Chaudhuri S 2006 Nanotechnology 171533

Fancher C A, de Clercq H L, Thomas O C, Robinson D W and Bowen K H 1998 J. Chem. Phys. 1098426

Gao P X and Wang Z L 2004 J. Phys. Chem. B108 7534

Greene L E, Law M, Goldberger J, Kim F, Johnson J C, Zhang Y, Saykally R J and Yang P 2003 Angew. Chem. Int. Ed. 42 3031

Greene L E, Law M, Tan D H, Montano M, Goldberger J, Somorjai G and Yang P 2005 Nano Lett. 51231

Guo L, Ji Y L, Xu H, Simon P and Wu Z 2002 J. Am. Chem. Soc. 12414864

Ham H, Shen G, Cho J H, Lee T J, Seo S H and Lee C J 2005 Chem. Phys. Lett. 40469

Hsu N E, Hung W K and Chen Y F 2004 J. Appl. Phys. 96 4671

Huang M H et al 2001 Science 2921897

Kar S, Pal B N, Chaudhuri S and Chakravorty D 2006 J. Phys. Chem. B110 4605

Liang J, Liu J, Xie Q, Bai S, Yu W and Qian Y 2005 J. Phys. Chem. B109 9463

Liao L, Li J C, Wang D F, Liu C S, Fu Q and Fan L X 2005 Nanotechnology 16985

Li Q, Kumar V, Li Y, Zhang H, Marks T J and Chang R P H 2005 Chem. Mater. 171001
Li S Y, Lin P, Lee C Y and Tseng T Y 2004 J. Appl. Phys. 95 3711

Li Y B, Bando Y and Golberg D 2004 Appl. Phys. Lett. 843603

Li Z, Ding Y, Xiong Y, Yang Q and Xie Y 2004 Chem. Eur. J. 105823

Lv R, Cao C, Guo Y and Zhu H 2004 J. Mater. Sci. 391575

Lyu S C, Zhang Y, Ruh H, Lee H J, Shim H W, Suh E K and Lee C J 2002 Chem. Phys. Lett. 363134

Lyu S C, Zhang Y, Lee C J, Ruh H and Lee H J 2003 Chem. Mater. 153294

Milne W L et al 2004 J. Mater. Chem. 14933

Nilsson L et al 2000 Appl. Phys. Lett. 762071

Pan Z W, Mahurin S M, Dai S and Lowndes D H 2005 Nano Lett. 5723

Park W I, Kim D H, Jung S W and Yi G C 2002 Appl. Phys. Lett. 804232

Roy V A L, Djurisic A B, Chan V, Gao J, Lui H F and Surya C 2003 Appl. Phys. Lett. 83141

Saito Y and Uemura S 2000 Carbon 38169

Senda S, Sakai Y, Mizuta Y, Kita S and Okuyama F 2004 Appl. Phys. Lett. 855679

Sun X M, Chen X, Deng Z and Li Y D 2002 Mater. Chem. Phys. 7899

Wang X D, Summers C J and Wang Z L 2004 Nano Lett. 4 423

Wang Z L 2004 Mater. Today 726

Xiong Y, Xie Y, Yang J, Zhang R, Wu C and Du G 2002 J. Mater. Chem. 123712

Yu H, Zhang Z, Han M, Hao X and Zhu F $2005 \mathrm{~J}$. Am. Chem. Soc. 1272378

Zhang J, Zhang S Y and Chen H Y 2004 Chem. Lett. 331054 